\title{
Transfusion of Ipses-Derived Leukocytes to Kill Cancer
}

\author{
Jiang $\mathrm{Li}^{1^{*}}$, Ying Cui ${ }^{2}$, Guodong Gao ${ }^{1^{*}}$, Ti-Fei Yuan \\ ${ }^{1}$ Department of Neurosurgery, Tang Du Hospital, The Fourth Military University, Xi' an, China; ${ }^{2}$ Department of Clinical Laboratory \\ and Research center of Tang Du Hospital, The Forth Military Medical University; Xi' an, China; ${ }^{*}$ Corresponding author \\ Email: lijiang19771103@gmail,gguodong@fmmu.edu.cn,ytf0707@126.com
}

Received October $28^{\text {th }}, 2009$; revised December $23^{\text {rd }}, 2009$; accepted January $23^{\text {rd }}, 2010$.

\begin{abstract}
Here we propose that the rejuvenation of leukocytes with iPSC technology in vitro and transfusion of cancer cellresistant white blood cells back to human body provide a prospective therapy for cancer patients.
\end{abstract}

Keywords: Ipscs, Leukocytes, Cancer

\section{Introduction}

60 years ago, Chester Southam injected healthy people and cancer patients with Hela cells, and found that healthy human beings are resistant to cancer cells, the ability of which was lost in cancer patients [1]. The important factors that lead to the loss of innate immunity during cancer progression include the failure of leukocytes (white blood cells) in killing cancer cells and the fusion of cancer cell and leukocyte, which results in the metastasis of cancer cells [2]. In recent years, a spontaneous cancer-resistant mice line was found, whose resistance is mediated by rapid infiltration of leukocytes and some other cells with innate immunity [3]. In these mice, the killing of injected cancer cells was completed by the migration of leukocytes to the cancer cells-injected site, the identification/recognition of specific antigens on the surface of cancer cells, as well as the release of cancer cell-killing substances, the first two steps of which do not exist in normal mice lines [3]. Additionally, aging leads to the reduction of this resistance ability, while repeated challenge with cancer cell injection can enhance the resistance during ageing periods. It is thus conceivable that the failed or weakened resistance of innate immunity cells, especially leukocytes, contribute to the cancer progress in patients.

To restore the innate immunity, efforts must be made to re-activate the activity of white blood cells in patients with cancer, or to replace those "sick" leukocytes with healthy, young leukocytes engineered in vitro, the process of which can now be completed with the recently emerged iPSC technology.

\section{IPSC Technology}

The regenerative medicine or rejuvenation therapy requires a large number of health cells to be placed in damaged parts of the body. However only limited sources of adult stem cells are available, and the self-repair ability in mammals including human is very restricted. The use of embryonic stem cells suffers from ethical difficulties, and the transplantation of heterogeneous tissues often meets immunological challenges. In the last two years, the successes of inducible pluripotent stem cell (iPSC) technology on human cells [4,5], including patientssourced cells [6], provide new hopes in generating immune-free stem cell lines in treating human patients.

iPSC can be acquired by transfection of somatic cell with pluripotent factors-coding viral vectors, or the direct delivery of DNA plasmids into cytoplasm to promote a pluripotent fate $[4,5]$. Recent studies found that the transient delivery of these proteins without genome integration can also bring pluripotency [7,8]. Additionally, numerous small molecules have been identified to promote the efficiency in generating iPSCs $[9,10]$, including natural compounds such as Vitamin C [11]. Methods with further improved safety and efficiency are expected in coming years.

The iPSCs, once stabilized, can be expanded greatly with many kinds of trophic factors and cytokines. Moreover, additional genetic engineering could be performed to repair potential genetic defects of the patients, or to promote the differentiation of iPSC into a defined fate. It will be therefore possible to acquire large number of cells, such as leukocytes, for repeated clinical use. 


\section{The Hypothesis}

We hypothesize that the transfusion of healthy, cancer cell-resistant leukocytes (in future, may include other innate immunity associated cells) generated with iPSC technology can be used to treat cancer patients. More importantly, genetic modified leukocytes (such as the knockout of specific receptors [12]) could be resistant to cancer cell fusion, therefore stopping the progression of cancer cells in vivo.

However it should be also noted that white blood cells family contains several types of cells such as neutrophils, eosinophils and basophils; these cells are of diverse function within the tumor microenvironment. For example, a recent study identified different subpopulations of neutrophils (N1-N2) that may inhibit or promote the tumor growth [13]. Therefore it will be useful to genetically engineer only a subpopulation of one kind of leukocytes in reaching effective tumor control.

\section{Testing the Hypothesis and Clinical Significance}

There're few technical difficulties to generate iPSCs from cancer patients and promote these cells into a leukocyte cell fate. The fibroblasts from patients' skin tissues could be most easily harvested and induced into iPSCs with well-built protocols, while theoretically all types of somatic cells from biopsies can be used. Some left questions are the efficiency in expanding the precursor cells and directed differentiation from iPSCs into leukocytes. These procedures could benefit from protocols inducing ESCs into leukocytes [14].

It will be useful to start with the mice model, to compare the efficiency of healthy leukocyte transfusion with other therapies in stopping cancer progression and metastasis. One question to be determined is to what extent the defected resistance of white blood cells contributes to the cancer cell progress in human patients. Should one kind of cells/subpopulation of cells or cellular signaling pathways be determined to drive tumor progression in humans, surely the new therapy with genetically engineered iPSC-derived white blood cell transfusion would follow.

\section{REFERENCES}

[1] A. E. Moore, C. P. Rhoads, and C. M. Southam, "Homotransplantation of human cell lines," Science, Vol. 125, No. 3239, pp. 158-160, 1957.

[2] J. M. Pawelek and A. K. Chakraborty, "The cancer cellleukocyte fusion theory of metastasis," Advances in Cancer Research, Vol. 101, pp. 397-444. 2008.

[3] A. M. Hicks, G. Riedlinger, M. C. Willingham, M. A.
Alexander-Miller, C. Von Kap-Herr, M. J. Pettenati, A. M. Sanders, H. M. Weir, W. Du, J. Kim, et al., "Transferable anticancer innate immunity in spontaneous regression/complete resistance mice," Proceedings of the National Academy of Science, USA, Vol. 103, No. 20, pp. 7753-7758, 2006.

[4] J. Yu, M. A. Vodyanik, K. Smuga-Otto, J. AntosiewiczBourget, J. L. Frane, S. Tian, J. Nie, G. A. Jonsdottir, V. Ruotti, R. Stewart, et al., "Induced pluripotent stem cell lines derived from human somatic cells," Science, Vol. 318, No. 5858, pp. 1917-1920, 2007.

[5] K. Takahashi, K. Tanabe, M. Ohnuki, M. Narita, T. Ichisaka, K. Tomoda, and S. Yamanaka, "Induction of pluripotent stem cells from adult human fibroblasts by defined factors," Cell, Vol. 131, No. 5, pp. 861-872, 2007.

[6] G. Amabile and A. Meissner, "Induced pluripotent stem cells: Current progress and potential for regenerative medicine," Trends in Molecular Medicine, Vol. 15, No. 2, pp. 59-68, 2009.

[7] D. Kim, C. H. Kim, J. I. Moon, Y. G. Chung, M. Y. Chang, B. S. Han, S. Ko, E. Yang, K. Y. Cha, R. Lanza, et al., "Generation of human induced pluripotent stem cells by direct delivery of reprogramming proteins," Cell Stem Cell, Vol. 4, No. 6, pp. 472-476, 2009.

[8] H. Zhou, S. Wu, J. Y. Joo, S. Zhu, D. W. Han, T. Lin, S. Trauger, G. Bien, S. Yao, Y. Zhu, et al., "Generation of induced pluripotent stem cells using recombinant proteins," Cell Stem Cell, Vol. 4, No. 5, pp. 381-384, 2009.

[9] T. Lin, R. Ambasudhan, X. Yuan, W. Li, S. Hilcove, R. Abujarour, X. Lin, H. S. Hahm, E. Hao, A. Hayek, et al., "A chemical platform for improved induction of human iPSCs," Nature Methods, Vol. 6, No. 11, pp. 805-808, 2009.

[10] Y. Shi, Y. ZHao, and H. Deng, "Powering reprogramming with vitamin C," Cell Stem Cell, Vol. 6, pp.1-2, 2010.

[11] M. A. Esteban, T. Wang, B. Qin, J. Yang, D. Qin, J. Cai, W. Li, Z. Weng, J. Chen, S. Ni, et al., "Vitamin C Enhances the Generation of Mouse and Human Induced Pluripotent Stem Cells," Cell Stem Cell, 2009.

[12] J. M. Pawelek and A. K. Chakraborty, "Fusion of tumour cells with bone marrow-derived cells: A unifying explanation for metastasis," Nature Reviews Cancer, Vol. 8, No. 5, pp. 377-386, 2008.

[13] Z. G. Fridlender, J. Sun, S. Kim, V. Kapoor, G. Cheng, L. Ling, G. S. Worthen, and S. M. Albelda, "Polarization of tumor-associated neutrophil phenotype by TGF-beta: "N1" versus "N2" TAN," Cancer Cell, Vol. 16, No. 3, pp. 183-194, 2009.

[14] M. Hannig, H. R. Figulla, H. Sauer, and M. Wartenberg, "Control of leukocyte differentiation from embryonic stem cells upon vasculogenesis and confrontation with tumour tissue," Journal of Cellular and Molecular Medicine, 2008. 